\title{
Die Einsprachigkeit des Anderen oder die ursprüngliche Prothese
}

JACQUES DERRIDA

- Denk Dir, stell‘ Dir jemanden vor, der mit dem Französischen einen kultivierten Umgang pflegte. Und der, wenn er auch noch französischer Staatsbürger wäre, also ein Subjekt - wie man sagt - der französischen Kultur darstellte. Nun fiele es diesem Subjekt der französischen Kultur eines Tages ein, in gutem Französisch zum Beispiel zu sagen: »Ich habe nur eine Sprache, und die ist nicht die meinige / die gehört nicht mir.« Und weiter oder auch: »Ich bin einsprachig. Meine Einsprachigkeit bleibt, und ich nenne sie meine Bleibe und empfinde sie als solche, ich bleibe dort und wohne in ihr. Sie wohnt in mir. Die Einsprachigkeit, in der ich sogar atme, ist für mich das Element. Nicht ein Naturelement, auch nicht die Transparenz des Äthers, aber ein absolutes Milieu; unüberschreitbar, unbestreitbar. Ich kann sie nur zurückweisen, indem ich ihre Allgegenwart in mir bestätige. Sie wird mir von jeher voraussein. Das bin ich. Diese Einsprachigkeit, für mich bin ich das. Was nicht heißen soll, auf keinen Fall, glaube das bloß nicht, daß ich eine allegorische Figur dieses Tieres oder dieser Wahrheit bin: der Einsprachigkeit. Aber außerhalb ihrer würde ich nicht ich selbst sein. Sie konstituiert mich, sie diktiert mir noch die Selbstheit [ipséité] von allem auf, sie schreibt mir auch eine mönchische Einsamkeit vor, als ob Gelübde mich gebunden hätten, noch bevor ich sprechen lernte. Dieser unerschöpfliche Solipsismus, das bin ich noch vor mir. Auf Dauer. Niemals wird also diese Sprache, die einzige, die ich unter diesen Umständen zu sprechen bestimmt bin, insofern mir im Leben und im Tod sprechen möglich ist, niemals - verstehst $\mathrm{Du}$ - wird diese einzige Sprache die meinige sein. Niemals war sie in Wahrheit die meinige. Du erkennst auf einen Schlag den Ursprung meiner Leiden, da diese Sprache sich ganz durch sie hindurchzieht, und den Ort meiner Passionen, meiner Begehrungen, meiner Bitten, die Bestimmung meiner Hoffnungen. Aber ich habe Unrecht, ich habe Unrecht, von Durchziehen oder von Ort zu reden. Denn es geschieht einzig und allein am Rande des Französischen, weder in ihm noch außerhalb seiner, sondern auf der unauf- 
findbaren Linie seines Randes, so daß ich mich - seit jeher, auf Dauer - frage, ob man in einer anderen Sprache - oder ohne jemandem etwas zu sagen, ohne überhaupt zu sprechen - lieben, genießen, bitten, vor Schmerz krepieren oder ganz einfach krepieren kann. Aber hier vor allem und zu allem Überfluß die Zweischneidigkeit einer scharfen Klinge, die ich Dir anvertrauen wollte, beinahe ohne ein Wort zu sagen; ich leide und ich genieße durch das, was ich Dir in unserer gemeinsam und alltäglich genannten Sprache sage: »Ja, ich habe nur eine Sprache, und die ist nicht die meinige."

- Du sprichst vom Unmöglichen. Deine Rede hat weder Hand noch Fuß. Sie bleibt immer zusammenhanglos, »inconsistent«, wie man im Englischen sagen würde. Auf jeden Fall dem Anschein nach inkonsistent, grundlos in ihrer aufscheinenden Eloquenz, denn ihre Redeweise [rhétorique] läßt den Sinn ins Unmögliche umschlagen. Dein Satz hat keinen Sinn, er hat keinen gesunden Menschenverstand, Du kannst sehen, wie er sich selbst aufhebt. Wie könnte jemand eine Sprache haben, die nicht die seinige wäre? Und vor allem, wenn man darauf besteht, und Du tust es, daß man nur eine, eine einzige, ganz allein habe? Du schiebst eine Art von feierlicher Beglaubigung vor, die sich dummerweise an den eigenen Haaren in einen logischen Widerspruch verwickelt. Was der Wissenschaftler vielleicht an einem so schlimmen Fall, der sich selbst für unheilbar hält, als noch schlimmer diagnostizieren würde: Dein Satz löscht sich selbst in einem logischen Widerspruch aus, der noch übertroffen wird von einem pragmatischen oder performativen Widerspruch. Es ist aussichtslos. Die performative Geste der Aussage vermag vielmehr als Akt das Gegenteil von dem zu beweisen, was das Zeugnis zu sagen behauptet, das heißt eine bestimmte Wahrheit. »Niemals war sie in Wahrheit [meine]«, wagtest Du zu sagen. Derjenige der spricht, das Subjekt der Aussage, $\mathrm{Du}$, aber ja, das Subjekt der französischen Sprache, das hört man das Gegenteil von dem tun, was es sagt. Es ist als ob Du lügen würdest, indem Du im selben Atemzug die Lüge gestehst. Eine Lüge, die nun unglaubhaft wird und die den Glauben in Deine Redeweise untergräbt. Die Lüge wird durch die Tatsache dessen dementiert, was sie als Sprechakt tut. Sie beweist so praktisch das Gegenteil von dem, was Deine Rede zu bejahen, zu beweisen, zu verifizieren behauptet. Man kann gar nicht mehr aufhören, Deine Absurdität bloßzustellen. - Nun gut, ich werde zunächst, bevor ich anfange, zwei Vorschläge riskieren. Auch sie werden unvereinbar [incompossible] scheinen, das heißt nicht nur in sich widersprüchlich, sondern diesmal auch untereinander widersprüchlich. Sie nehmen die Form eines Gesetzes an, es gibt beidemal ein Gesetz. Den antagonistischen Bezug, den die beiden Gesetze jedesmal aufeinander haben, kannst Du also, wenn Dir das Wort gefällt, das ich liebe, Antinomie nennen:

1. Man spricht immer nur eine einzige Sprache.

2. Man spricht niemals eine einzige Sprache.

Dieser zweite Vorschlag geht in Richtung dessen, was mein Freund Khatibi in der Präsentation seines Werks über die Zweisprachigkeit in genau dem Moment klar zum Ausdruck bringt, in dem er summa summarum eine Problematik und ein Programm umgrenzt. Ich rufe ihn also zu Hilfe: 
Wenn es (wie wir es im Anschluß und zusammen mit anderen sagen) die Sprache nicht gibt, wenn es keine absolute Einsprachigkeit gibt, dann bleibt einzukreisen, was eine Muttersprache in ihrer aktiven Teilung ist und was zwischen dieser Sprache und der sogenannten Fremdsprache übertragen/aufgepfropft wird. Was dabei übertragen/aufgepfropft wird und was dabei verloren geht, wobei es weder der einen noch der anderen zugute kommt: das Nichtmitteilbare. Von der Doppel-Sprache [bi-langue] in ihren Auswirkungen auf Sprechen und Schreiben [...]. ${ }^{1}$

»Teilung« [division], sagt er. »Aktive Teilung«. Aus dem Grund schreibt man, träumt man vielleicht zu schreiben. Und aus diesem Grunde sind es eher zwei Motivationen als eine, ein einziger Grund, aber ein Grund, der von der sogenannten »Teilung « durchzogen ist, aus diesem Grunde erinnert man sich beim Tun immer, beunruhigt man sich, macht man sich auf die Suche nach Geschichte und nach Filiation. An diesem Ort der Eifersucht, an diesem von Rache und Ressentiment geteilten Ort, in diesem von seiner eigenen »Teilung « erregten Körper wird, vor aller anderen Form von Gedächtnis, die Schrift wie von selbst der Anamnese zugedacht. Selbst wenn sie vergißt, ruft sie noch dieses Gedächtnis in Erinnerung, nennt sie sich so, Schrift, nennt sie sich aus dem Gedächtnis. Ein blinder genealogischer Trieb findet gerade in der Aufteilung dieses doppelten Gesetzes, in der antinomischen Duplizität dieser Zugehörigkeitsklausel seinen Antrieb, seine Kraft und seine Zuflucht:

1. Man spricht immer nur eine einzige Sprache - oder vielmehr ein einziges Idiom.

2. Man spricht niemals eine einzige Sprache - oder vielmehr, es gibt kein reines Idiom.

- Wäre es also möglich? Du bittest mich, Dir aufs Wort zu glauben. Und Du hast gerade »Idiom« der »Sprache« hinzugefügt. Das ändert gar manches. Eine Sprache ist kein Idiom, und das Idiom ist auch kein Dialekt.

- Mir ist die Notwendigkeit dieser Unterscheidungen nicht unbekannt. Linguisten und generell Wissenschaftler mögen gute Gründe haben, sich daran zu halten. Ich glaube gleichwohl nicht, daß sie in aller Strenge und bis an die äußerste Grenze aufrecht erhalten werden können. Wenn man in einem stets sehr eingegrenzten Kontext externe Kriterien nicht in Betracht zieht, seien sie nun »quantitativ« (Angestammtheit, Stabilität, demographische Ausdehnung des Felds der Rede [parole]) oder »symbolisch-politisch « (Legitimität, Autorität, Vorherrschaft einer »Sprache« [langue] über eine Redeweise, einen Dialekt oder ein Idiom), dann weiß ich nicht, wo man interne und strukturelle Züge finden kann, um zwischen Sprache, Dialekt und Idiom streng zu unterscheiden. Auf jeden Fall, selbst wenn das, was ich hier sage, problematisch bliebe, würde ich immer noch diesen Standpunkt einnehmen, hinsichtlich dessen diese Unterscheidung noch in der Schwebe bleibt - zumindest durch Übereinkunft zwischen uns und vorläufig. Denn die Phänomene, die mich interessieren, sind gerade diejenigen, denen es gelingt, diese Grenzziehungen zu verwirren, sie zu überschreiten und

1 | Abdelkebir Khatibi: Du bilinguisme. Paris 1985, S. 10. 
folglich ihre historische Künstlichkeit ebenso wie ihre Gewaltsamkeit sichtbar werden zu lassen, das heißt die Kräfteverhältnisse, die sich dort konzentrieren und sich in Wahrheit unabsehbar kapitalisieren. Diejenigen, die zum Beispiel sensibel für all die Spielarten der »Kreolisierung« sind, ermessen es besser als andere.

Formulieren wir also eine Hypothese und lassen wir sie arbeiten. Nehmen wir an, daß ich Abdelkebir Khatibi - ohne ihn verletzen zu wollen - eines Tages beim Kolloquium in Louisiana, fern von seinem Zuhause und fern von meinem, von unserem Zuhause, eine Eröffnung durch die treue und bewundernde Zuneigung hindurch mache, die ich für ihn empfinde. Was sollte ihm diese öffentliche Erklärung erklären? Ungefähr folgendes: »Lieber Abdelkebir, schau, ich halte mich hier für den am meisten franko-maghrebinischen von uns beiden und vielleicht sogar für den einzigen Franko-Maghrebiner. Wenn ich mich irre, wenn ich mich täusche oder wenn ich übertreibe, nun gut, dann bin ich sicher, daß man mir widersprechen wird. Ich werde dann versuchen, mich zu erklären oder mich so gut zu rechtfertigen, wie ich kann. Schauen wir uns um und klassifizieren wir, teilen wir auf und gehen wir nach Gruppierungen vor.

A. Es gibt unter uns frankophone Franzosen, die keine Maghrebiner sind: Franzosen aus Frankreich, mit einem Wort französische Staatsbürger, die aus Frankreich gekommen sind.

B. Es gibt unter uns auch >Frankophone<, die weder Franzosen noch Maghrebiner sind: Schweizer, Kanadier, Belgier oder Afrikaner aus verschiedenen Ländern Zentralafrikas.

C. Es gibt schließlich unter uns frankophone Maghrebiner, die keine Franzosen sind und die nie Franzosen im Sinne von französischen Staatsbürgern waren: Du zum Beispiel und andere Marrokaner oder Tunesier.

Wie Du also siehst, gehöre ich keiner der klar abgegrenzten Gruppen an. Meine >Identität< stammt von keiner der drei Kategorien ab. Wo soll ich mich also einordnen? Und welche Taxonomie soll ich erfinden? Meine Hypothese lautet daher, daß ich hier vielleicht allein, der alleinige bin, der von sich sagen kann, daß er gleichzeitig maghrebinisch (das ist keine Staatsangehörigkeit) und französischer Staatsbürger ist. Zugleich der/das eine und der/das andere. Und mehr noch zugleich das eine und das andere durch Geburt. Denn ist die Geburt, die Nationalität durch Geburt, die eingeborene Kultur hier nicht unser Thema? (Eines Tages wird man ein anderes Kolloquium der Nationalsprache [langue], der Nationalität, der kulturellen Zugehörigkeit diesmal durch den Tod, durch den Grabkult widmen und mit dem Geheimnis des Ödipus auf Kolonos beginnen müssen: der ganzen Macht, die dieser >Fremde< über die >Fremden < hinsichtlich des Allergeheimsten des Geheimnisses seiner letzten Ruhestätte innehat, ein Geheimnis, das er wahrt oder der Obhut des Theseus anvertraut im Tausch gegen das Heil der Stadt und der kommenden Generationen, ein Geheimnis, das er dennoch seinen Töchtern verweigert, um ihnen so selbst ihre Tränen und eine angemessene >Trauerarbeit $<\mathrm{zu}$ nehmen). Aber sind wir nicht übereinge- 
kommen, daß wir hier von der sogenannten Muttersprache und von Geburt bezüglich des Bodens, von Geburt bezüglich des Blutes und - was etwas ganz anderes bedeutet - von Geburt in bezug auf die Sprache sprechen? Und von den Bezügen zwischen Geburt, Sprache, Kultur, Nationalität und Staatsbürgerschaft? Jedenfalls lautete meine Hypothese, daß mein >Fall zu keiner der drei oben angeführten Gruppen gehört. Und wäre das nicht auch die einzige Rechtfertigung meiner Anwesenheit - sofern sie denn eine ist - auf diesem Kolloquium?« Ungefähr das hätte ich Abdelkebir Khatibi zu Anfang erklärt. Was Du in diesem Moment geneigt sein mögest zu hören, das ist jedenfalls die Geschichte, die ich mir erzähle, die ich mir gerne erzählen möchte oder die ich vielleicht im Namen [titre] des Zeichens, der Schrift und der Anamnese, als Antwort auch auf den Titel dieses Treffens, den Titel der Renvois d'ailleurs [Verweisungen nach anderswo] oder der Echoes from elsewhere [Echos von anderswoher], wohl auf eine kleine Fabel zurückführe. Auch wenn ich das Gefühl geäußert habe, daß ich hier und dort der einzige Franko-Maghrebiner bin, so autorisiert mich das nicht, im Namen von irgend jemandem und vor allem nicht im Namen irgendeiner franko-maghrebinischen Wesenheit zu sprechen, deren Identität gerade in Frage steht. Wir kommen erst dazu, denn all das ist in meinem Fall noch weit davon entfernt, klar zu sein. Unsere Frage gilt immer der Identität. Was ist Identität, dieser Begriff, dessen transparente Identität mit sich selbst in so vielen Debatten über Mono- oder Multikulturalismus, über Nationalität, Staatsbürgerschaft und Zugehörigkeit im allgemeinen immer dogmatisch vorausgesetzt wird? Und noch bevor es um die Identität des Subjekts geht, was ist Selbstheit? Diese beschränkt sich nicht auf die abstrakte Fähigkeit, »ich« zu sagen, der sie immer schon vorausgegangen sein wird. Sie bedeutet vielleicht in erster Linie die Macht eines »ich kann«, das ursprünglicher ist als das »ich« in einer Kette, in der das »pse« von ipse sich nicht mehr von der Macht trennen läßt, von der Meisterschaft oder der Souveränität des hospes (ich beziehe mich hier auf die semantische Kette, die im Innern sowohl der Gastfreundschaft wie der Feindschaft arbeitet - hostis, hospes, hosti-pet, posis, despotes, potere, potis sum, possum, pote est, potest, pot sedere, possidere, compos, etc. -). ${ }^{2}$ Franko-Maghrebiner zu sein, es »wie ich « zu sein, das bedeutet - im besonderen wie im allgemeinen - kein Übermaß oder keinen Überfluß an Identitäten, Attributen oder Namen. All das würde vielmehr und sogleich eine Störung der Identität verraten. Erkenne an diesem Ausdruck »Störung der Identität« das ganze Gewicht, ohne die psychopathologischen oder sozio-pathologischen Konnotationen auszuschließen. Um mich als Franko-Maghrebiner zu präsentieren, spiele ich auf die Staatsbürgerschaft an. Die Staatsbürgerschaft definiert aber, wie man weiß, keine kulturelle, sprachliche oder historische Zugehörigkeit im allgemeinen. Sie deckt nicht all

2 | Es handelt sich dabei um eine Kette, die Benveniste bekanntlich an verschiedenen Stellen rekonstruiert und herausgestellt hat, vor allem in einem großartigen Kapitel, das der "Gastfreundschaft" gewidmet ist (in: Le Vocabulaire des institutions indo-européennes. Paris 1969, Bd. 1, S. 87 ff.), ein Kapitel, auf das ich vielleicht anderswo auf eine mehr problematisierende oder beunruhigende Weise zurückkommen werde. 
diese Zugehörigkeiten ab. Aber sie ist auch nicht einfach eine äußerliche oder dem Überbau zugehörige Eigenschaft, die an der Oberfläche der Erfahrung dahingleitet. Vor allem dann nicht, wenn diese Staatsbürgerschaft noch ganz und gar unsicher, frisch, bedroht und artifizieller denn je ist. Das ist es in »meinem Fall«, das ist die zugleich typische und einzigartige Situation, von der ich sprechen wollte. Vor allem wenn man diese Staatsbürgerschaft erst im Laufe seines Lebens erworben hat, was vielleicht zahlreichen Amerikanern passiert ist, die hier bei diesem Kolloquium anwesend sind, aber auch wenn man sie zunächst im Laufe seines Lebens verloren hat, was sicherlich kaum je einem Amerikaner passiert ist. Und wenn eines Tages irgendein Individuum erlebt hat, daß ihm die Staatsbürgerschaft selbst entzogen wurde (was mehr als ein Paß, eine »Greencard «, ein passives oder aktives Wahlrecht ist), ist das denn jemals einer Gruppe als solcher passiert? Ich spiele hier wohlverstanden nicht auf diese oder jene ethnische Gruppe an, die sich absonderte, sich eines Tages von einem anderen Nationalstaat befreite oder eine Staatsbürgerschaft aufkündigte, um sich eine andere in einem ganz neu etablierten Staatsgebilde zu verleihen. Für diese Umwälzung gibt es genug Beispiele. Nein, ich spreche von einer »gemeinschaftlichen« Gruppierung (einer »Masse«, die zehn- oder hunderttausende von Personen als Gruppe zusammenschließt), von einer »ethnisch« oder »religiös « gedachten Gruppe, die als solche sich eines Tages ihrer Staatsbürgerschaft durch einen Staat beraubt sieht, der sie ihr mit der Brutalität einer einseitigen Entscheidung entzieht, ohne sie um ihre Meinung zu fragen und ohne da $\beta$ die sogenannte Gruppe irgendeine andere Staatsbürgerschaft erhielte. Keine andere. Ich habe das erlebt. Zusammen mit anderen habe ich die französische Staatsbürgerschaft verloren und dann wiedererhalten. Ich habe sie für Jahre verloren, ohne eine andere zu haben. Nicht die geringste Staatszugehörigkeit, verstehst Du.

Ich hatte um nichts gebeten. Ich habe im Augenblick, als man sie mir nahm, kaum etwas von ihr gewußt, jedenfalls in der legalen und objektiven Form des Wissens, in der ich es hier ausbreite (denn ich habe wohl auf andere Weise von ihr gewußt, nun denn). Und dann, eines Tages, eines »schönen Tages« habe ich, ohne daß ich einmal mehr um etwas gebeten hätte, und noch zu jung, um etwas darüber in der Art eines eigentlich politischen Wissens zu wissen, die sogenannte Staatsbürgerschaft wiedererlangt. Der Staat, mit dem ich niemals gesprochen habe, hatte sie mir zurückerstattet. Der Staat, der nicht mehr der »Französische Staat« Petains war, erkannte mich erneut an. Das war 1943, glaube ich, ich war noch nicht »in Frankreich« gewesen, ich hatte mich noch niemals dorthin begeben. Eine Staatsbürgerschaft wächst im wesentlichen nicht einfach so, sie ist nicht natürlich. Aber das Artifizielle und Prekäre an ihr kommen besser, wie durch den Blitz einer privilegierten Offenbarung, zum Vorschein, wenn sich die Staatsbürgerschaft ins Gedächtnis ihrer noch frischen Erwerbung einschreibt: zum Beispiel der französischen Staatsbürgerschaft, die den algerischen Juden durch den Cremieux-Erlaß 1870 verliehen wurde; oder auch wenn es sich in das traumatische Gedächtnis einer »Entehrung«, eines Verlustes der Staatsbür- 
gerschaft einschreibt: zum Beispiel der Verlust der französischen Staatsbürgerschaft für die gleichen algerischen Juden weniger als ein Jahrhundert danach. So war es denn auch der Fall »unter der Besatzung«, wie man sagt. Ja, so »wie man sagt«, denn in Wirklichkeit ist es eine Legende. Algerien ist nie besetzt worden. Ich will sagen, daß es, wenn es je besetzt war, dann gewiß nicht durch deutsche Besatzer. Der Entzug der französischen Staatsbürgerschaft für die algerischen Juden war mit all seinen Folgen allein eine Tat der Franzosen. Sie haben das ganz allein entschieden nach eigenem Gutdünken, sie mußten davon schon seit langem geträumt haben und sie haben es ganz allein zustande gebracht. Ich war zu diesem Zeitpunkt sehr jung, ich verstand zweifellos nicht genau - schon damals verstand ich es nicht genau -, was Staatsbürgerschaft und der Verlust der Staatsbürgerschaft bedeutete. Aber ich zweifle nicht daran, daß der Ausschluß - zum Beispiel von der den jungen Franzosen zugesicherten Schulbildung - im Zusammenhang mit den Störungen der Identität, von denen ich Dir vorhin erzählte, stehen könnte. Und ich zweifle auch nicht daran, daß solche »Ausschlüsse« ihr Mal auf jener Zugehörigkeit oder Nicht-Zugehörigkeit von Sprache, auf jenem Anschluß an Sprache, auf jener Zuweisung an das, was man schlicht Sprache nennt, hinterlassen. Aber wer beherrscht sie denn genaugenommen? Und wen beherrscht sie? Hat sie, die Sprache, jemals etwas in Besitz, als beherrschenden oder besessenen Besitz? $\mathrm{Zu}$ eigen besessen oder besitzend, als ein Eigentum? Was hat es auf sich mit jenem Zuhause-Sein in der Sprache, auf das wir unaufhörlich zurückkommen?

Ich habe es gerade betont, die Aberkennung der Staatsbürgerschaft dauerte zwei Jahre, aber sie fand stricto sensu nicht »unter der Besatzung « statt. Es war eine innerfranzösische Operation; man sollte sogar sagen ein Akt des französischen Algeriens in Abwesenheit jeglicher deutscher Besatzung. In Algerien hatte man nie eine deutsche Uniform gesehen. Es gibt kein Alibi, kein Abstreiten, keine mögliche Täuschung: Es war unmöglich, die Verantwortung für diesen Ausschluß auf eine fremde Besatzung abzuschieben. Wir waren Geiseln der Franzosen, auf Dauer, denn etwas davon ist mir geblieben, soviel ich auch auf Reisen gehen mag. Und ich wiederhole: ich weiß nicht, ob es andere Beispiele in der Geschichte der modernen National-Staaten gibt, Beispiele für eine solche Beraubung der Staatsbürgerschaft, die für zigtausend Personen auf einmal verfügt wurde. Von Oktober 1940 an, der Aufhebung des Crémieux-Dekrets vom 24. Oktober 1870, verweigerte Frankreich selbst, der französische Staat in Algerien, der im Anschluß an einen parlamentarischen Akt, wie man weiß, legal (durch die Kammer der Volksfront!) gegründete »Französische Staat«, die französische Identität, vielmehr nahm er sie Menschen, deren kollektives Gedächtnis sich noch daran erinnerte oder kaum vergessen hatte, daß die Staatsbürgerschaft ihnen vor kurzem erst verliehen worden war und weniger als ein halbes Jahrhundert zuvor (1898) prompt zu mörderischen Verfolgungen und zum Beginn von Pogromen geführt hatte, was trotzdem eine »Assimilation« ohnegleichen nicht verhinderte: eine profunde, rasche, eifrige und spektakuläre Assimilation innerhalb zweier Generationen. Begünstigt nun diese Störung der Identität die Anamnese oder behindert sie sie? Schärft sie das Begehren nach 
Erinnerung oder enttäuscht sie das genealogische Phantasma? Unterdrückt, verdrängt oder befreit sie? Zweifellos alles zugleich, und das wäre noch eine andere Version, der andere Abhang des Widerspruchs, der uns in Bewegung versetzt; und der uns laufen läßt, bis wir außer Atem kommen oder den Kopf verlieren.

Unter dem Titel: »Die Einsprachigkeit des Anderen« stellen wir uns etwas figürlich vor, entwerfen wir eine Figur. Sie wird nur eine vage Ähnlichkeit sowohl mit mir selbst als auch mit der Gattung der autobiographischen Anamnese haben, die immer mit aller Strenge auftaucht, wenn man sich im Raum der Erzählung [relation] exponiert. Ich verstehe »relation« sowohl im Sinne von Narration, zum Beispiel der genealogischen Erzählung, als auch allgemeiner in dem Sinne, den Edouard Glissant diesem Begriff aufprägt, wenn er von einer Poetique de la Relation spricht, so wie man auch von einer Politik der Relation/Beziehung sprechen könnte. Ich wage es also, mich Dir, das ecce homo parodierend, als exemplarischen Franko-Maghrebiner zu präsentieren, aber auf entwaffnete Weise mit mehr naiven, weniger kontrollierten und weniger höflichen Akzenten. Ecce homo, denn es handelt sich sehr wohl um eine »Passion«, die man nicht belächeln sollte, um das Martyrium des Franko-Maghrebiners, der schon bei Geburt, seit seiner Geburt aber durch seine Geburt, auf der anderen Seite, der seinen, im Grunde genommen nichts gewählt und nichts erfaßt hat und der immer noch leidet und bezeugt. Was diese rätselhafte Bedeutung des Bezeugens beziehungsweise des exemplarischen Charakters des Beweises anbelangt, so haben wir es hier mit einer ersten und sicherlich allgemeinsten Frage zu tun: Was passiert, wenn jemand darauf verfällt, eine angeblich einzigartige Situation - zum Beispiel die meine und zwar indem er Zeugnis davon ablegt - in Ausdrücken zu beschreiben, die zu hoch für ihn und in einer Sprache formuliert sind, deren Allgemeingültigkeit einen gewissermaßen strukturellen, universellen, transzendentalen oder ontologischen Wert annimmt? Wenn der erste beste darunter versteht: »Was für mich gilt, gilt uneingeschränkt für alle. Die Substitution hat bereits begonnen, sie hat schon Wirkungen gezeitigt, jedermann kann für sich und von sich dasselbe sagen. Er reicht aus, mich zu verstehen, ich bin die universale Geisel.« [...]

Dem gängigen Verständnis nach setzt die autobiographische Anamnese die Identifizierung voraus. Genau genommen nicht die Identität. Eine Identität ist nie gegeben, empfangen oder erlangt, nein, allein der unbeendbare, unbestimmt phantasmatische Prozeß der Indentifizierung wird erduldet. Welche Geschichte einer Heimkehr zu sich oder nach Hause [chez soi], ins »Häuschen « [case] des Zuhause (chez kommt von casa) auch immer es sei, was auch immer es auf sich hat mit einer Odyssee oder einem Bildungsroman [dt. im Orig.], auf welche Weise auch immer eine Konstitution des Selbst, des autos, des ipse sich zurechtfabelt: immer stellt man sich vor, daß der- oder diejenige, die schreibt, bereits »ich« sagen können muß. Auf jeden Fall muß die identifikatorische Modalität schon oder fortan sichergestellt sein: und zwar sichergestellt durch die 
Sprache und in der Sprache. Man denkt, daß die Frage der Einheit der Sprache gelöst und das Eine der Sprache im strengen oder weiteren Sinne gegeben sein muß - ein weiter Sinn, den man so weit ausdehnen kann, bis alle identifikatorischen Modelle und Modalitäten, alle Pole imaginärer Projektion der Sozialkultur miteinbegriffen sind. Jeder Bereich findet sich hier in der Konfiguration mitrepräsentiert: die Politik, die Religion, die Künste, die Poesie und die schöngeistige Literatur, die Literatur im engeren (modernen) Sinne. Man muß schon wissen, in welcher Sprache ich gesagt wird, ich mich ausspreche. Man denkt hier sowohl an das ich denke als auch an das grammatikalische oder linguistische Ich, an das Ich oder an das Wir in ihrem identifikatorischen Status, so wie er von kulturellen, symbolischen und soziokulturellen Figuren gebildet wird. Man weiß sehr wohl, daß von all diesen Gesichtspunkten aus,die nicht allein grammatikalischer, logischer oder philosophischer Natur sind, das Ich der sogenannten autobiographischen Anamnese, das Ich-mich des ich erinnere mich sich je nach Sprache verschieden produziert und äußert. Es geht ihnen niemals vorher, es ist also nicht unabhängig von der Sprache im allgemeinen. Das ist wohlbekannt, aber selten von denen in Erwägung gezogen worden, die sich mit der Autobiographie im allgemeinen beschäftigen - egal ob diese Gattung nun literarisch ist oder nicht, ob man sie übrigens für eine Gattung hält oder nicht. Ohne uns nun hier auf die abgründigen Dinge einzulassen, die Sie ahnen, sollten wir uns vielleicht an eine einzige Konsequenz halten. Sie betrifft das, was unser Gemeinplatz während des Kolloquiums war, und zwar bereits von seinem Titel her: das heißt das Anderswo [ailleurs] und die Verweisung [renvoi], angenommen, sie könnten jemals einen Gemeinplatz ausmachen. Das in Frage stehende Ich hat sich sicherlich gebildet, man kann daran glauben, wenigstens wenn es dies hat tun können und wenn die Identitätsstörung, von der wir gerade sprachen, nämlich die Konstitution des Ich, die Bildung des Ich-Sagens, des Ich-mich oder die Erscheinung als solche einer prä-egologischen Selbstheit nicht im engeren Sinne betrifft. Es hätte sich also dieses Ich in einer unauffindbaren Situation gebildet, wobei es immer anderswohin, auf anderes, eine andere Sprache, den anderen im allgemeinen verweisen würde. Es hätte sich in einer nicht situierbaren Erfahrung der Sprache, der Sprache also im weiteren Sinne dieses Wortes, situiert. Diese Erfahrung war weder einsprachig noch zweisprachig oder mehrsprachig. Die Sprache war weder eine noch zwei, noch zwei $+n$. Auf jeden Fall gab es vor dieser befremdlicherweise vertrauten und eigentlich uneigentlichen (uncanny, unheimlichen [dt. im Orig.]) Situation einer unzählbaren Sprache kein denkbares oder denkendes Ich. Es ist unmöglich, die Sprachen abzuzählen, das wollte ich nahelegen. Es gibt keine Berechenbarkeit, wenn das Eine einer Sprache, die jeder arithmetischen Abzählbarkeit entgeht, niemals bestimmt ist. Das Eine der Einsprache, von der ich spreche, und diejenige, die ich spreche, stellen also keine arithmetische Identität dar, nicht einmal einfach eine Identität. Die Einsprache bleibt also unberechenbar, zumindest in dieser Hinsicht. Aber daß die Sprachen streng genommen überabzählbar scheinen, hindert sie alle nicht daran zu verschwinden. Sie gehen in diesem Jahrhundert zu Hunderten jeden Tag unter, und dieses Verderben eröffnet die 
Frage nach einer anderen Rettung oder einem anderen Heil [salut]. Wie kann man eine Sprache anders retten als dadurch, daß man Idiome archiviert (was wir manchmal auf wissenschaftliche, wenn nicht gar auf ausreichende Weise mit einer zunehmend anwachsenden Dringlichkeit tun)? Eine lebendige und »wohlbehaltene « Sprache? Was soll man von dieser neuen Soteriologie denken? Ist das gut? In welchem Namen? Und wenn es besser wäre, auf die Sprache zu verzichten, zumindest auf die besten Bedingungen des Überlebens »um jeden Preis « für ein Idiom zu verzichten, um Menschen zu retten, die in ihrer Sprache untergehen, um diese Menschen selbst zu erlösen, mit Ausnahme ihrer Sprache? Und wenn es besser wäre, Menschen zu retten als ihr Idiom, da, wo man wählen müßte? Denn wir leben in einer Zeit, wo sich diese Frage mitunter stellt. Auf der Erde von heute müssen gewisse Menschen der Homo-Hegemonie dominanter Sprachen weichen und die Sprache der Herren, des Kapitals und der Maschinen erlernen, müssen sie ihr Idiom verlieren, um zu überleben oder um besser zu leben. Es ist eine tragische Ökonomie, jeder Rat ist unmöglich. Ich weiß nicht, ob die Begrüßung [salut] des anderen das Heil [salut] des Idioms voraussetzt. Wir werden darüber noch sprechen, ebenso wie über dieses befremdliche französische Wort: salut.

Fahren wir also fort. Das, was ich sage, derjenige, den ich ausspreche, dieses Ich, von dem ich in einem Wort spreche, ist jemand, wie ich mich ungefähr erinnere, dem der Zugang zu jeder nichtfranzösischen Sprache Algeriens (arabischer Dialekt oder Literatur, Berberdialekt etc.) untersagt worden ist. Aber dieses selbe Ich ist auch jemand, dem der Zugang zum Französischen auf eine andere, anscheinend abwegige und perverse Art und Weise ebenfalls untersagt worden ist. Auf eine andere Weise, sicherlich, aber gleichermaßen untersagt. Durch ein Verbot, das auf einen Schlag den Zugang zu den Identifikationen untersagt, die eine friedliche Auto-Biographie, das heißt »Memoiren« im klassischen Sinne erlauben. In welcher Sprache soll man denn seine Memoiren schreiben, wenn es keine autorisierte Muttersprache gibt? Wie soll man ein »ich erinnere mich« aussprechen, das Geltung hat, wenn man sowohl die Sprache als auch sein Ich erfinden muß, sie gleichzeitig erfinden muß, jenseits dieses Aufbrandens [déferlement] von Amnesie, das vom doppelten Verbot entfesselt wurde? [...]

Denn nie konnte ich das Französische, diese Sprache, in der ich zu Dir spreche, »meine Muttersprache« nennen. Diese Worte kommen mir nicht in den Mund, sie kommen mir nicht aus dem Mund. Sie ist für die anderen »meine Muttersprache«. Das ist meine Kultur, sie hat mich die Katastrophen gelehrt, in die eine beschwörende Anrufung der Muttersprache die Menschen wohl gestürzt hat. Meine Kultur war von Beginn an eine politische Kultur. »Meine Muttersprache«, so sagen sie, so sprechen sie, ich zitiere sie und befrage sie. Ich frage sie - natürlich in ihrer Sprache, damit sie mich verstehen, denn das ist wichtig -, ob sie genau wissen, was sie sagen und wovon sie sprechen. Vor allem wenn sie so leichtfertig die »Verbrüderung « feiern, denn im Grunde ist es dasselbe Problem, die Brüder, die Muttersprache etc. Es ist fast so, als ob ich davon träumen würde, sie aufzuwecken, um ihnen zu sagen: »Hört zu, aufgepaßt, jetzt reicht es, ihr müßt aufstehen und davongehen, sonst geschieht euch 
ein Unglück oder - was aufs gleiche hinausläuft - es geschieht für euch nichts. Abgesehen vom Tod Eurer Muttersprache, was ihr so nennt, ihr werdet sehen, eines Tages wird sie euch nicht mehr antworten. Los, aufgebrochen jetzt. Hört zu ... glaubt nicht so schnell, glaubt mir, daß ihr ein Volk seid, hört auf, denen ohne Protest zuzuhören, die euch sagen >hört $\mathrm{zu}<\ldots \ll$.

- Abdelkebir Khatibi selbst spricht von seiner »Muttersprache«. Zweifellos ist es nicht das Französische, aber er spricht davon. Er spricht davon in einer anderen Sprache, nämlich dem Französischen. Er macht diese vertrauliche Mitteilung öffentlich, er publiziert seine Rede in unserer Sprache, und zwar um von seiner Muttersprache zu sagen - das ist die vertrauliche Mitteilung -, daß er sie »verloren« hat. Ja, mein Freund, zögere nicht, dann »meine Muttersprache« zu sagen. Er spricht davon nicht ohne Zittern, man kann es hören, nicht ohne jenes diskrete Beben der Sprache, das die poetische Vibration seines ganzen Werkes auszeichnet. Aber er scheint nicht vor Worten wie »Muttersprache« zurückzuweichen. Das ist das Vertrauen, das ich in dieser vertraulichen Mitteilung wiederfinde. Er bejaht sogar - was noch etwas anderes ist - die Besitzanzeige. Jedenfalls wagt er das, er bejaht sich selbst als Besitzenden, als ob sich kein Zweifel in seine Drohung hier einschleichen würde: »meine Muttersprache«, sagt er. Das sticht hervor, sicherlich unauffällig und in aller Stille, aber es sticht hervor. Das Hervorstechende dieses Zuges unterscheidet gerade die Geschichte, die ich erzähle, die Fabel, die ich von mir erzähle, die Verwicklung, deren Repräsentant, deren Zeuge, andere würden vielleicht zu schnell sagen: deren Kläger ich hier bin. Das Hervorstechende dieses Zuges unterscheidet sie von der Erfahrung, die Khatibi beschreibt, wenn er den Anruf der Schrift hört. Man glaubt schon, ihn zu hören, wie er dem Anruf in dem Moment lauscht, in dem dieser erklingt. Er erreicht ihn als Echo, er kommt auf ihn zu als Widerhall einer Doppelsprache [bi-langue]. Khatibi hält das zungenfertige Tritonshorn [conque volubile] einer doppelten Sprache gegen sein Ohr. Aber seit, ja seit dem Aufschlagen jenes großen Buches Amour bilingue [Zweisprachige Liebe] gibt es eine Mutter, eine einzige. Und was für eine Mutter: Derjenige, der in der ersten Person spricht, erhebt seine Stimme ausgehend von der Sprache seiner Mutter her. Er beschwört eine Ursprungssprache, die ihn vielleicht hat »verlorengehen [perdu] lassen«, aber die er nicht verloren [perdue] hat. Er bewahrt, was ihn hat verlorengehen lassen. Und er bewahrte natürlich auch schon, was er nicht verloren hat. Als ob er dessen Heil sichern könnte, und sei es durch seinen eigenen Untergang. Er hatte eine Mutter und zweifellos mehr als eine Mutter, aber er hat wohl auch seine Muttersprache, eine Muttersprache, eine einzige Muttersprache plus eine andere Sprache gehabt. Er kann also sagen: »meine Muttersprache«, ohne an der Oberfläche die geringste Verwirrung erscheinen zu lassen:

$\mathrm{Ja}$, meine Muttersprache hat mich verlorengehen lassen. Verloren? Ach was, sprach ich nicht, schrieb ich nicht in meiner Muttersprache mit großem Genuß? Und war die Zwei-Sprachigkeit nicht meine Chance des Exorzismus? Ich möchte etwas anderes damit sagen. Meine Mutter war Analphabetin. Meine Tante - meine Amme - war es auch. 
Eine angeborene Diglossie hat mich vielleicht der Schrift geweiht, zwischen dem Buch meines Gottes und meiner Fremdsprache, durch sekundenlange sekundäre Geburtsschmerzen jenseits jeder Mutter, der einen und der einzigen. Als Kind rief ich die Tante anstelle der Mutter, die Mutter anstelle des anderen, für immer den anderen, den anderen. $^{3}$

Obwohl meine eigene Mutter an schwierigen Tagen ihrer letzten Lebensjahre beinahe aphasisch und gedächtnislos wurde; obwohl sie sogar meinen Namen vergessen zu haben schien, war sie zweifellos keine Analphabetin. Aber im Gegensatz zu der Tradition, in der Khatibi zur Welt kam, sprach meine Mutter ebensowenig wie ich - ich habe es weiter oben angedeutet - eine Sprache, die man »im vollen Sinne« Muttersprache nennen könnte. Versuchen wir also fortan, die Dinge direkter zu bezeichnen, auch auf das Risiko hin, sie falsch zu benennen.

Erstens, das Verbot. Ein Verbot - halten wir dieses Wort provisorisch fest ein besonderes Verbot wurde also, ich erinnere, über die arabischen Sprachen und die Berbersprachen verhängt. Es nahm wohl für jemanden meiner Generation kulturelle und soziale Formen an, aber zuerst einmal war es eine schulische Sache, eine Sache, die einem »in der Schule « passierte, wobei es sich kaum um eine Maßnahme oder eine Entscheidung, sondern vielmehr um ein pädagogisches Dispositiv handelte. Das Verbot hatte seinen Ursprung in einem »Erziehungssystem «, wie man in Frankreich seit einiger Zeit, ohne Witz und ohne Beunruhigung, sagt. In Anbetracht all der kolonialen Zensurmaßnahmen - vor allem in den städtischen oder vorstädtischen Gebieten, in denen ich lebte -, in Anbetracht der sozialen Trennwände, der Rassismen einer Fremdenfeindlichkeit mit manchmal bedrohlich verzerrtem und manchmal wohlwollend »lebenslustigem «, manchmal sogar einladendem oder fröhlichem Antlitz, in Anbetracht des zunehmenden Verschwindens des Arabischen als offizieller, alltäglicher und administrativer Sprache, war die Schule die einzige Zuflucht und dort das Erlernen des Arabischen, aber als Fremdsprache, als befremdliche Art von Fremdsprache als Sprache des anderen freilich, aber gleichwohl - das war das Fremde und Beunruhigende - auch des anderen als ganz nahen Nächsten. Unheimlich [dt. im Orig.]. Für mich war es die Sprache des Nachbarn, denn ich wohnte am Rande eines arabischen Viertels, an einer der zugleich unsichtbaren und beinahe unüberschreitbaren nächtlichen Grenzen: die Segregation war dabei ebenso wirksam wie subtil. Ich muß hier auf die Feinanalysen verzichten, die eine Sozialgeographie des Wohnens ebenso wie Kartographie der Klassenräume in der Grundschule verlangen würden, wo es noch - bevor sie an der Schwelle zum Gymnasium verschwanden - viele kleine Algerier, Araber und Kabyle gab. Ganz nahe und unendlich fern, so war die Distanz, deren Erfahrung man uns sozusagen einschärfte, eine unvergeßliche und verallgemeinerbare Erfahrung. Das fakultative Lernen des Arabischen war freilich erlaubt. Wir wußten, daß es gestattet war, das heißt jedoch, alles andere als gefördert wurde. Die

3 | Khatibi: Du bilinguisme (Anm. 1), S. 75. 
Autoritäten der nationalen Erziehung (der »öffentlichen Ausbildung«) behandelten es unter dem gleichen Titel, im gleichen Atemzug und in der gleichen Form wie irgendeine andere Fremdsprache in allen französischen Gymnasien Algeriens. Das Arabische als fakultative Fremdsprache in Algerien! Als ob man uns sagen würde, und das sagte man uns eigentlich: »Mal sehen, Latein ist Pflichtfach für alle in der Sechsten, natürlich, und sprechen wir nicht alle Französisch, aber wollt ihr zu allem Überfluß auch noch Englisch, Arabisch, Spanisch oder Deutsch lernen?« Niemals jedoch die Berbersprache, scheint mir. Ohne jetzt Statistiken zur Hand zu haben, erinnere ich mich, daß der Prozentsatz der Gymnasiasten, die das Arabische wählten, gegen Null ging. Durch ihre extreme Seltenheit bildeten diejenigen nicht einmal eine homogene Gruppe, die sich infolge ihrer Wahl darauf einließen, was damals als ungewöhnlich oder bizarr erschien. Unter ihnen gab es nur manchmal Schüler algerischer Abstammung (»Eingeborene«, wie sie offiziell hießen), wenn sie ausnahmsweise ins Gymnasium kamen - denn damals wandten sich nicht alle unter ihnen von selbst dem Arabisch als Sprachfach zu. Unter denen, die sich für das Arabische entschieden, gab es - glaube ich - kleine Algerienfranzosen nicht-städtischer Herkunft, die Söhne von Siedlern, die aus »dem Innern« kamen. Dem Rat und dem Wunsch ihrer Eltern folgend, wobei Not kein Gebot kennt, glaubten sie im voraus, das Arabische aus technischen und beruflichen Gründen eines Tages zu benötigen: unter anderem, damit ihre Feldarbeiter sie verstanden, und das heißt auch, ihnen zuhörten, und in Wirklichkeit, ihnen gehorchten. All die anderen, zu denen auch ich gehörte, nahmen das Verbot passiv hin. Dieses stellte den massiven Grund ebenso wie die Wirkung - eine gesuchte Wirkung also der zunehmenden Bedeutungslosigkeit, der organisierten Marginalisierung dieser Sprachen des Arabischen und des Berberischen dar. Ihre Schwächung wurde von einer kolonialen Politik kalkuliert, die so tat, als ob sie Algerien als den Zusammenschluß von drei französischen Departements behandelte. Ich kann hier diese Politik der Sprache nicht frontal analysieren, und ich möchte mich des Wortes »Kolonialismus« nicht zu leichtfertig bedienen. Jede Kultur ist ursprünglich kolonial. Wir müssen nicht allein auf die Etymologie zählen, um dies in Erinnerung zu rufen. Jede Kultur wird durch die einseitige Auferlegung irgendeiner »Politik« der Sprache eingesetzt. Die Herrschaft beginnt, wie man weiß, mit der Macht zu benennen, seine Benennungen aufzuerlegen und zu rechtfertigen. Man weiß, wie es mit dem Französischen in Frankreich selbst war, im revolutionären Frankreich ebenso oder mehr noch als im monarchistischen Frankreich. Diese souveräne Mahnung [mise en demeure] kann offen, legal, bewaffnet oder auch listenreich sein, versteckt unter den Alibis des »universellen« Humanismus oder bisweilen der freigebigsten Gastfreundschaft. Sie folgt der Kultur immer oder geht ihr voraus wie ihr Schatten. Es geht nicht darum, die arrogante Besonderheit und die traumatisierende Brutalität dessen wegzuwischen, was man den modernen Kolonialkrieg im »eigentlichen Sinne« nennt, ob im Augenblick selbst der militärischen Eroberung oder wenn die symbolische Eroberung den Krieg mit anderen Mitteln fortsetzt. Im Gegenteil. Die koloniale Grausamkeit haben einige, darunter auch ich, sozusagen auf 
beiden Seiten erfahren. Aber immer offenbart sie auf exemplarische Weise auch dort die koloniale Struktur aller Kultur. Sie zeugt davon als Märtyrer und auf heftige Weise [à vif].

Die Einsprachigkeit des Anderen wäre zunächst diese Souveränität, dieses Gesetz, das zweifellos von anderswoher gekommen ist, aber auch und zunächst die Sprache selbst des Gesetzes und das Gesetz als Sprache. Seine Erfahrung wäre offensichtlich autonom, denn ich muß über dieses Gesetz reden und es mir aneignen, um es zu verstehen, $a l s$ ob ich es mir selbst geben würde. Aber es bleibt notwendigerweise - so will es das Wesen eines jeden Gesetzes im Grunde - heteronom. Der Wahnsinn des Gesetzes siedelt seine Möglichkeit auf Dauer im Herd dieser Auto-Heteronomie an. Die vom anderen auferlegte Einsprachigkeit funktioniert, indem sie auf diesem Grund Fuß faßt und zwar hier durch eine Souveränität, die im Wesen immer kolonialistisch ist und die dahin tendiert - unterdrückt oder nicht -, die Sprachen auf das Eine, das heißt die Hegemonie des Homogenen zu reduzieren. Man findet es überall bestätigt, überall, wo diese Homo-Hegemonie in der Kultur am Werk ist, wobei sie die Falten glättet und den Text abflacht. Die kolonisierende Gewalt selbst hat es im Grunde genommen nicht nötig, dafür spektakuläre Initiativen anzuregen: religiöse Missionen, gute philanthropische oder humanitäre Werke, Marktuntersuchungen, militärische oder völkermordende Expeditionen.

Man wird mir vorwerfen, daß ich das alles durcheinanderwerfe. Aber nein! Aber doch, man kann und man darf nicht - bei aller Beachtung der allerstrengsten Unterscheidungen, bei aller Respektierung der Achtung des Ehrwürdigen - diese obskure gemeinsame Stärke, diesen kolonialistischen Trieb aus den Augen verlieren, der damit begonnen haben wird, sich in das einzuschleichen wobei er niemals zögern würde, darüber herzufallen -, was sie mit einem bis zur Geistlosigkeit abgenutzten Ausdruck: »die Beziehung zum anderen!« oder »die Öffnung für den anderen!« nennen. Aber gerade aus diesem Grunde bedeutet die Einsprachigkeit des anderen auch noch etwas anderes, wie sich nach und nach herausstellen wird: nämlich daß man nur eine Sprache spricht - und daß man sie nicht hat. Man spricht von jeher nur eine Sprache - und sie ist auf asymmetrische Weise, so daß sie immer dem anderen zukommt, einem vom anderen her wiederkehrt, vom anderen bewahrt wird. Sie ist vom anderen gekommen, beim anderen geblieben und zum anderen zurückgekehrt. Natürlich konnte der einmal blockierte Zugang zur Sprache und Schrift eines solchen anderen - hier des Arabers und des Berbers - ebenso wie zur ganzen, davon untrennbaren Kultur, konnte die Einschreibung dieser Grenze nicht spurlos bleiben. Sie sollte vor allem Symptome einer Faszination bei der scheinbar gemeinsamen und privilegierten Praxis des Französischen vervielfältigen. Die unterschlagene Sprache - das Arabische und Beberische, um damit anzufangen - wurde die allerfremdeste Sprache. Aber dieses Privileg war mit einer eigenartigen und beunruhigenden Nähe verbunden. Manchmal frage ich mich, ob diese unbekannte Sprache nicht meine bevorzugte Sprache ist, und zwar die erste unter meinen bevorzugten Sprachen. Und so wie bei jeder meiner bevorzugten Sprachen (denn ich gestehe, daß ich mehr als eine habe) möchte ich sie 
vor allem außerhalb jeder »Kommunikation « in der poetischen Feierlichkeit des Gesangs oder des Gebets vernehmen. Es wird mir fortan um so schwerer fallen zu zeigen, daß die französische Sprache uns gleichermaßen verboten war. Und zwar gleichermaßen, aber ich muß einräumen: anders.

Zweitens, das Verbotene. Ich wiederhole es, auch diese Erfahrung wurde wieder und vor allem durch die Schule vermittelt. Man kann darin eine Geschichte von Lehre und Unterricht erkennen, aber von schulischer Lehre und schulischem Unterricht. Ein solches Phänomen mußte sich auf mehrere Ebenen von Allgemeingültigkeit verteilen. Es umkreiste Kreise soziolinguistischer Abschließung, die zugleich exzentrisch und konzentrisch waren. Für die Schüler der französischen Schule in Algerien, ob sie nun gebürtige Algerier, »französische Landesangehörige «, 4 »französische Staatsbürger Algeriens« oder ob sie im Milieu der Juden in Algerien geboren waren, die nacheinander sowohl das eine als auch das andere waren (»eingeborene Juden«, wie man unter der Besatzung ohne Besatzung sagte, eingeboren und trotzdem für einige Zeit französisch) für alle war das Französische angeblich eine Muttersprache, deren Quelle, deren Normen, deren Regeln, deren Gesetze jedoch woanders angesiedelt waren. Sie waren woandershin verwiesen, würden wir sagen, um den Titel unseres Kolloquiums wieder ins Spiel zu bringen oder umzukehren. Anderswohin, das bedeutete in die Metropole, in die Haupt-Mutter-Vater-Stadt. Man sagte bisweilen »Frankreich«, aber häufiger die Metropole, jedenfalls in der offiziellen Sprache, der von Reden, Zeitungen und Schulen auferlegten Redeweise. In meiner Familie und beinahe überall sagte man unter sich »Frankreich « (»die da können sich Ferien in Frankreich leisten«, »der da wird in Frankreich studieren«, »er wird nach Frankreich in die Kur fahren, im allgemeinen nach Vichy«, »dieser Lehrer kommt aus Frankreich«, »dieser Käse kommt aus Frankreich«). Die Metropole, die Haupt-Mutter-Vater-Stadt, die City der Muttersprache, hier haben wir einen Ort, der, ohne es zu sein, ein fernes Land darstellte, nah aber dennoch weit entfernt, nicht fremd - das wäre zu einfach -, aber fremdartig, phantastisch und phantomartig. Im Grunde genommen frage ich mich, ob nicht eine meiner ersten und imposantesten Figuren des Gespenstischen, des Gespenstischen selbst, Frankreich war, das heißt alles, was diesen Namen trug (vorausgesetzt, daß ein Land und das, was den Namen eines anderen trägt, niemals etwas anderes sind, selbst für die unverdächtigsten Patrioten und vielleicht vor allem für sie). Es war also ein Traumland, in einer nicht objektivierbaren Entfernung. Als Modell des Wohl-Sprechens und Wohl-Schreibens repräsentierte es die Sprache

4 | Zu diesem juristischen Ausdruck wie zur außergewöhnlichen Geschichte der Staatsbürgerschaft in Algerien (zu der es meines Wissens keine Entsprechung stricto sensu in der Welt gibt) verweise ich auf den erhellenden Artikel von Louis-Augustin Barriere: "Le puzzle de la citoyennté en Algerie", erschienen in der Revue du Gisti (Plein droit Nr. 29/30, November 1995), dessen heute exemplarische Arbeit ich bei dieser Gelegenheit begrüßen möchte. Dieser Artikel beginnt folgendermaßen (aber man muß alles lesen): "Bis zur Befreiung wurden die Moslems Algeriens nur als französische Landesangehörige und nicht als französische Staatsbürger betrachtet. Diese Unterscheidung erklärte sich durch die Geschichte." 
der Herren (ich glaube übrigens, daß ich keinen anderen Souverän in meinem Leben anerkannt habe). Der Herr erschien zunächst und besonders in Gestalt des Schullehrers. Dieser konnte so auf würdige Weise und in der universellen Gestalt der guten Republik den Herrn im allgemeinen repräsentieren. Ganz anders als für den kleinen Franzosen aus Frankreich war die Metropole ein Anderswo, das heißt zugleich ein gesicherter Platz und ein ganz anderer Ort. Seit der unersetzlichen Verortung dieses mythischen Dortdrüben mußte man - natürlich vergeblich - versuchen, die unendliche Distanz oder die maßlose Nähe des unsichtbaren aber strahlenden Mittel Punktes zu bemessen, von dem alle Paradigmen der Unterscheidung, der Verbesserung, der Eleganz und der literarischen und oratorischen Sprache zu uns gelangten. Die Sprache der Metropole war die Muttersprache, in Wahrheit jedoch das Surrogat einer Muttersprache (gibt es jemals etwas anderes?) als Sprache des anderen. Für den kleinen Provenzalen oder den kleinen Bretonen gibt es natürlich ein analoges Phänomen. Paris kann immer diese Rolle der metropolis zusichern und diesen Platz für einen Provinzler einnehmen, so wie es die vornehmen Viertel für eine gewisse Art von Banlieue tun. Paris, das ist auch die Hauptstadt der Literatur. Aber in diesem Fall hat der Andere nicht mehr die gleiche Transzendenz des Dortdrüben, die Ferne des Anderswoseins, die unerreichbare Autorität eines Herren, der in Übersee wohnt. Es fehlt ganz einfach das Meer. Denn wir wußten es auf dunkle, aber gewisse Art und Weise, daß Algerien keineswegs die Provinz war noch Algier ein Arbeiterviertel. Für uns war Algerien seit der Jugend auch ein Land, Algier eine Stadt in einem Land in einem zweifelhaften Sinn des Wortes, das weder mit Staat noch mit Nation, noch mit Religion, auch nicht, wie ich zu sagen wage, mit authentischer Gemeinschaft übereinstimmte. Und in diesem »Land « Algerien sah man übrigens, wie sich das gespenstische Trugbild einer Struktur von Hauptstadt vs. Provinz wieder etablierte (»Algier vs. Landes innere«, »Algier vs. Oran«, »Algier vs. Constantine«, »Algier-Stadt vs. AlgierVorort«, vornehme Viertel, generell auf den Hügeln vs. Armenviertel meistens weiter unten). 